\title{
Environmental water chemistry impacts silver nanoparticle characteristics, interactions with microbiota, and mechanisms of toxicity
}

\author{
Janice Speshock ${ }^{1 *}$, Everest Castaneda ${ }^{1}$, Laura K Braydich-Stolle ${ }^{2}$, Elizabeth M Maurer ${ }^{3}$ and Jeff A Brady \\ ${ }^{1}$ Department of Biological Sciences, Tarleton State University, 1333 W. Washington Street, Stephenville, TX 76401 USA \\ ${ }^{2}$ Human Effectiveness Directorate, United States Air Force Research Laboratories, Wright-Patterson Air Force Base, OH 45433, USA \\ ${ }^{3}$ American Testing Services, Miamisburg, OH 45342, USA \\ ${ }^{4}$ Texas A\&M AgriLife Research and Extension Center, Stephenville, TX 76401, USA
}

\begin{abstract}
It has been well established that synthesis methods can cause variability in nanotoxicity studies, but here we demonstrate that environment can play a role as well. Silver nanoparticles were dispersed in two different environmentally-obtained fresh water samples for an ecotoxicological assessment of their impact on zebrafish. The two water sources varied in alkalinity, $\mathrm{pH}$, and total hardness, and this variation impacted nanoparticle agglomeration size, uptake into the zebrafish kidneys, inflammation and apoptosis, and the zebrafish microbiota.
\end{abstract}

\section{Introduction}

Silver nanoparticles (AgNPs) have many practical purposes in science and medicine, thus their use has expanded exponentially over the past decade [1]. Many laboratories are attempting to synthesize different sizes, shapes, and compositions of AgNPs to develop useful medical devices or novel antimicrobials [1,2]. However, the toxicity of AgNPs is just beginning to be understood. AgNPs can be toxic at high doses, but they tend to dissociate into $\mathrm{Ag}^{+}$ions even at lower doses, and these ions are capable of interacting with many cellular structures, especially proteins, causing cellular dysfunction [2]. AgNPs have been demonstrated to interact with many types of cells: animal, bacterial, fungal, protozoal, and plant; and often internalize into the cells to elicit their effects as ions $[1,3]$. They can induce reactive oxygen species (ROS), alter mitochondrial function, interfere with ATP synthesis, and interact with transcription factors to impair cell signaling [2-4]. These effects result in the non-specific cell death of both prokaryotic and eukaryotic cells [3].

The volume of AgNPs being synthesized today creates an environmental pollution concern [1]. The failure to regulate manufacturing and consumer waste disposal can lead to incidental or purposeful administration of AgNPs into the environment, which ultimately end up interacting with organisms in the aquatic ecosystem [1]. AgNPs can be highly toxic to aquatic organisms due to the same mechanisms of toxicity for terrestrial species [3]. Marine invertebrates and small fish make good indicators of aquatic toxicity due to their small size and high-degree of susceptibility to environmental pollutants $[3,5]$. Zebrafish (Danio rerio) are a model organism for aquatic studies since the amount of available materials and data on these fish make them efficient for streamlining ecotoxicological testing [5].
Single exposures of $10 \mathrm{~nm}$ AgNPs at non-toxic doses are sufficient to disturb the normal physiology of zebrafish following an intramuscular injection [6]. However, zebrafish are not naturally injected by AgNPs, but would likely absorb them through their gills or skin. Here we wanted to determine if the zebrafish are capable of internalizing low doses of AgNPs from various natural water sources, and if those internalized AgNPs impacted cellular function. Zebrafish were placed into two different natural water sources that were exposed to 5 micrograms per milliliter $(\mu \mathrm{g} / \mathrm{mL})$ of $40 \mathrm{~nm}$ AgNPs and their inflammatory response, caspase activity, and core microbiome were assessed for changes in the presence of a low dose administration of AgNPs.

\section{Methods}

\section{Water sources}

Two environmental freshwater sources were used for comparing the effects of silver nanoparticles on zebrafish. Water was collected from the Bosque River in the City Park of Stephenville, Texas, and from a pond at the local high school in Stephenville, TX. The water chemistry was compared using a 6-in-1 aquarium test strip (Tetra Melle, Germany) before initiating the experiments, and the total hardness, total alkalinity, and $\mathrm{pH}$ were much greater in the river water than in the pond water (Table 1). These water samples were used for the experiments described below.

${ }^{*}$ Correspondence to: Speshock J, Associate Professor, Department of Biological Sciences, Tarleton State University, 1333 W. Washington Street, Stephenville, TX 76401 USA, Tel: [254] 968-9341; E-mail: speshock@tarleton.edu

Key words: water hardness, metagenomics, inflammation, caspases, apoptosis

Received: November 08, 2019; Accepted: January 13, 2020; Published: January 16,2020 
Table 1. Comparison of water chemistry between the river and pond water sources

\begin{tabular}{|c|c|c|}
\hline & River & Pond \\
\hline Nitrate $(\mathrm{mg} / \mathrm{L})$ & $<20$ & $<20$ \\
\hline Nitrite $(\mathrm{mg} / \mathrm{L})$ & 0.5 & 0 \\
\hline Total Hardness $(\mathrm{ppm})$ & 300 & 150 \\
\hline Chlorine $(\mathrm{ppm})$ & 0.5 & 0.5 \\
\hline Total alkalinity $(\mathrm{ppm})$ & 300 & 180 \\
\hline $\mathrm{pH}$ & 8.4 & 7.4 \\
\hline
\end{tabular}

Table 2. Zebrafish mortality following a 24-hour exposure to river and pond water with or without $5 \mu \mathrm{g} / \mathrm{mL}$ of $40 \mathrm{~nm}$ AgNPs

\begin{tabular}{|c|c|c|}
\hline & Fish inoculated & Fish survived \\
\hline River-AgNP & 16 & $16(100 \%)$ \\
\hline River+AgNP & 16 & $12(75 \%)$ \\
\hline Pond-AgNP & 16 & $16(100 \%)$ \\
\hline Pond+AgNP & 16 & $16(100 \%)$ \\
\hline
\end{tabular}

\section{Fish exposures}

Zebrafish were purchased and shipped from Doctor Foster and Smith (Rhinelander, WI) and were housed in an aquarium with $26^{\circ} \mathrm{C}$ water, 14/10-hour light/dark cycles, fed once daily. Twenty-four hours before nanoparticle exposure, the fish were moved to sterile containers with holes in the lids, 2 fish per container, with $25 \mathrm{~mL}$ of either river or pond water to acclimate ( $n=32$ per water source). Following the acclimation period, biopure $40 \mathrm{~nm}$ AgNPs (nanoComposix, San Diego, CA) were added to half of the sterile containers of both pond and river water with fish ( $\mathrm{n}=16$ per water source) at 5 micrograms per $\mathrm{mL}$ and were stirred in with a sterile pipet tip. The fish were placed into an incubator at $26^{\circ} \mathrm{C}$ for 24 hours. The physical health of the fish was assessed, which indicated that 4 fish died in the river water with AgNPs, but no distress was observed in any of the other groups (Table 2 ). The fish were euthanized in an ice slush and were necropsied for their organs for use in the subsequent experiments. All procedures were approved by the TSU IACUC AUP030614.

\section{Nanoparticle characterization}

To understand the state of the nanoparticles in the environmental water samples, dynamic light scattering (DLS) and UV-Vis spectroscopy were performed on the nanoparticles in the river and pond water samples as well as those that were added to deionized water or the aquarium housing water in the same concentration $(5 \mu \mathrm{g} / \mathrm{mL})$ for comparison. One $\mathrm{mL}$ of each water sample was removed and analyzed using a Zetasizer Nano-ZS (Malvern Instruments, United Kingdom) to determine the size of the nanoparticles, and a NanoDrop 2000 UV-Vis spectrometer (Thermo Fisher Scientific, Waltham, MA) to determine particle integrity.

\section{Silver levels}

Kidneys were extracted from 4 fish per treatment. The kidney tissue was homogenized and processed for inductively coupled plasma mass spectrometry (ICP-MS) as per previously described methods [6].

\section{Gene expression}

RNA was isolated from 4 fish per treatment using the Aurum Total RNA mini kit (Biorad, Hercules, CA) with optional DNase treatment, and quantified using the Qubit RNA HS Assay kit (Thermo Fisher Scientific, Waltham, MA) on a Qubit fluorometer. cDNA was synthesized from $100 \mathrm{ng}$ of total RNA using the iScript cDNA synthesis kit (Biorad, Hercules, CA). Quantitative PCR analysis was performed on the pro-inflammatory cytokines (interleukin-1 beta (IL-1 $\beta$ ), interleukin-6 (IL-6), and tumor necrosis factor alpha (TNF- $\alpha$ )) and caspases 6 and 9 using a previously described technique and primers [6]. The critical point was determined via the software on the Roche 480 lightcycler (Basel, Switzerland) following 40 amplification cycles. The pond water samples were compared with and without AgNPs, with the nanoparticle-free pond water serving as a control. The river water samples were compared with and without AgNPs, with the nanoparticle-free river water serving as a control. The fold change was calculated using the previously described delta-delta Ct method [7].

\section{Microbial Community Analysis}

DNA was extracted from the intestines and the skin from the left side of the fish between the pectoral and caudal fins using a previously described technique [8]. Bacterial communities were amplified with primers 341F 5'-CCTACGGGNGGCWGCAG-3' and 805R (GACTACHVGGGTATCTAATCC) that target bacterial V3 and V4 regions of the $16 \mathrm{~S}$ ribosomal RNA locus [9]. Paired-end sequence data were generated on an Illumina MiSeq instrument using v3 600 cycle kits (Illumina, San Diego, CA), with dual 6 bp dual index sequences attached to each amplicon during indexing PCR as described in the Illumina 16S Metagenomic Sequencing Library Preparation protocol [10]. PCR conditions included initial denaturation at $95^{\circ} \mathrm{C}$ for $3 \mathrm{~min}$, amplification with 35 cycles of: $95{ }^{\circ} \mathrm{C}$ for 10 s, $55^{\circ} \mathrm{C}$ for 30 s and 72 ${ }^{\circ} \mathrm{C}$ for 30 s. The raw sequencing reads were processed with QIIME and USEARCH software [11,12]. Sequence tags were compared to the Greengenes 13.8 reference sequence database [13] using UCLUST [12] in order to pick referenced-based Operational Taxonomic Units (OTUs) at $97 \%$ similarity and taxonomy assignment was carried out with RDP [14]. Random subsampling of sequences from each sample without replacement was done to provide even measures of microbial alpha- and beta-diversity and to have equal sequencing depth ( 854 sequences/sample) for the production of all figures, tables, and statistical analyses.

\section{Statistical analyses}

DLS, ICP-MS, and qRT-PCR levels were compared experimental (with AgNPs) to control (no AgNPs) using a Student's t-test (significance occurring at $\mathrm{P}<0.05$ ). Microbial community shifts following nanoparticle treatments were determined using unweighted unifrac distance matrices [15]. Sequence counts of taxonomic groupings were compared between samples with non-parametric t-tests, while Chaol species richness and Shannon species diversity metrics were compared between samples using t-tests.

\section{Results}

\section{Agglomeration of AgNPs}

The AgNPs purchased had a mean diameter of $40 \pm 4 \mathrm{~nm}$ according to the manufacturer and only a slight increase in size was observed in deionized (DI) $\mathrm{H}_{2} \mathrm{O}$ according to DLS measurements, with a mean size of $48.8+/-0.1$ (Figure 1). A much greater agglomeration effect was observed when the AgNPs were added to the river or pond water with mean sizes of $188+/-9.4$ and $248+/-81.6$, respectively (Figure 1).

\section{Stability of AgNPs}

UV-Vis spectroscopy illustrated a characteristic peak around the wavelength of $410 \mathrm{~nm}$, confirming that AgNPs were in the exposed water samples, but not in the water where no silver was added (Figure 2). A breakdown in the peak was observed when AgNPs were added to other water samples besides deionized $\mathrm{H} 2 \mathrm{O}$, suggesting an instability of the nanoparticles in these environmental water samples leading to $\mathrm{Ag}^{+}$ 


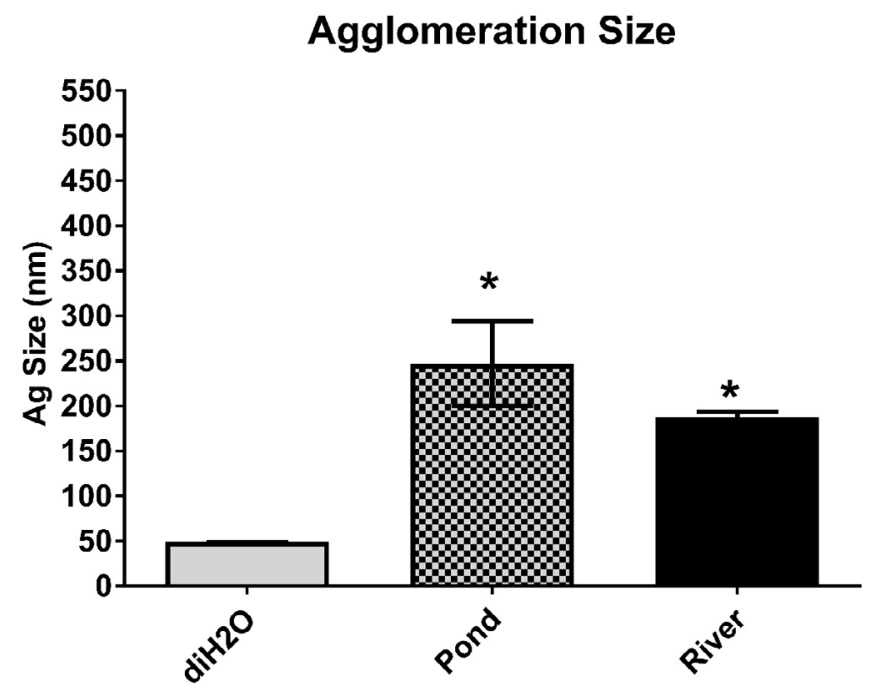

Figure 1. Size of AgNPs in solution. 50 micrograms per milliliter of $40 \mathrm{~nm}$ AgNPs were added to $1 \mathrm{~mL}$ of river, pond, or deionized (control) water, and dynamic light scattering was used to determine the average size in solution. *indicates $P<0.001$

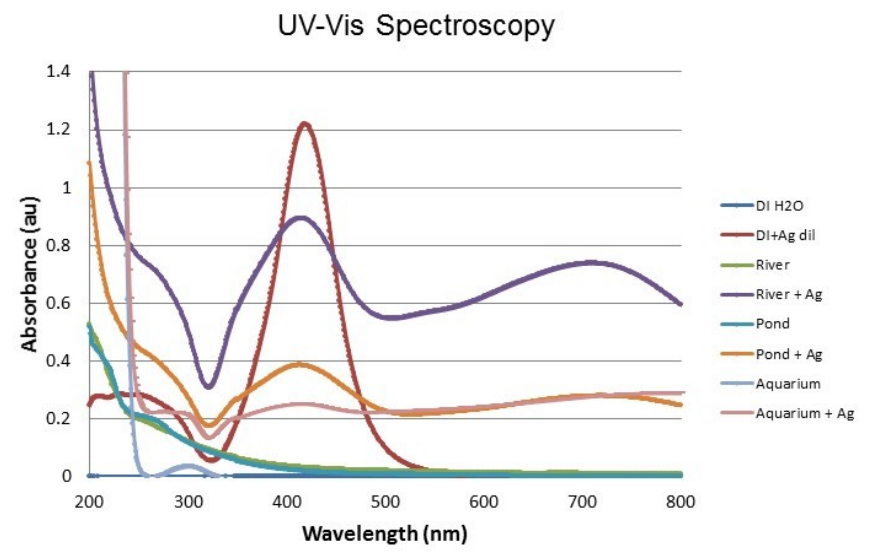

Figure 2. Confirmation of silver peak. A UV-Vis spectrum was analyzed for each water sample with and without the addition of 5 micrograms $/ \mathrm{mL}$ of $40 \mathrm{~nm}$ AgNPs. The characteristic silver peak at $410 \mathrm{~nm}$ was observed in all samples that had the addition of AgNPs and was absent in all control samples

ion dissociation, especially in the pond and aquarium water samples (Figure 2). The Ag peak measured at 1.2 absorbance units (AU) for those AgNPs dissolved in the deionized $\mathrm{H}_{2} \mathrm{O}$, whereas those dissolved in the pond and river waters had $\mathrm{AU}$ measurements of 0.4 and 0.9, respectively (Figure 2).

\section{Uptake of AgNPs}

Since the kidneys are involved in the filtration of the blood, and they were observed to be a major target of AgNPs in a previous study [6], we examined the amount of silver that entered the kidney following environmental exposure to $40 \mathrm{~nm}$ AgNPs. There was no detectable silver in the kidneys of untreated fish (data not shown), but in the fish exposed to AgNPs in river water or pond water had $250+/-3.47 \mathrm{ng}$ and $75+/-0.52 \mathrm{ng}$ of silver, respectively, as determined through ICP-MS of processed kidneys (Figure 3).

\section{Gene expression changes by AgNPs}

There was little change in the pro-inflammatory cytokine gene expression in the kidneys of the fish from the river water samples between the control river and Ag-treated river, with the exception of a slight, but significant, decline in TNFa expression (Figure 4). There was also no increase in caspase 9 gene expression in the AgNP treated fish in the river water (Figure 4). However, there was a significant 15.86fold (+/- 4.35) increase in caspase 6 expression in the Ag-river fish compared to the fish in the control river water (Figure 4).

Addition of AgNPs to the pond water significantly increased the expression of both interleukins (IL)-1 and -6 in the fish kidneys, with a $7.05(+/-2.09)$ and $8.09(+/-2.41)$-fold increases, respectively, over

\section{Inductively-Coupled Plasma Mass Spectrometry}

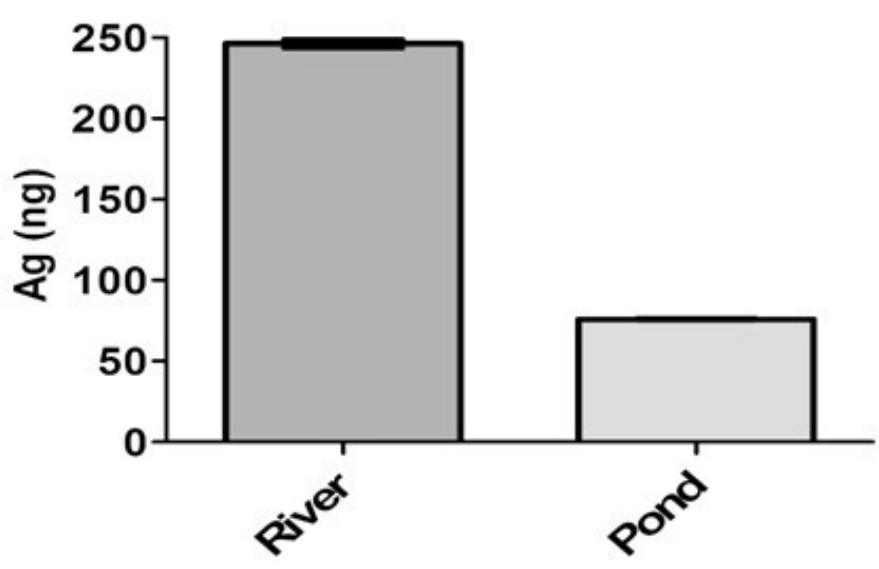

Figure 3. Silver levels in the kidneys of the zebrafish. ICP-MS was used to measure the amount of silver detectable in the kidneys of exposed zebrafish. There was no silver detected in the kidneys of zebrafish that did not receive AgNP treatment

\section{Quantitative Real-Time PCR}

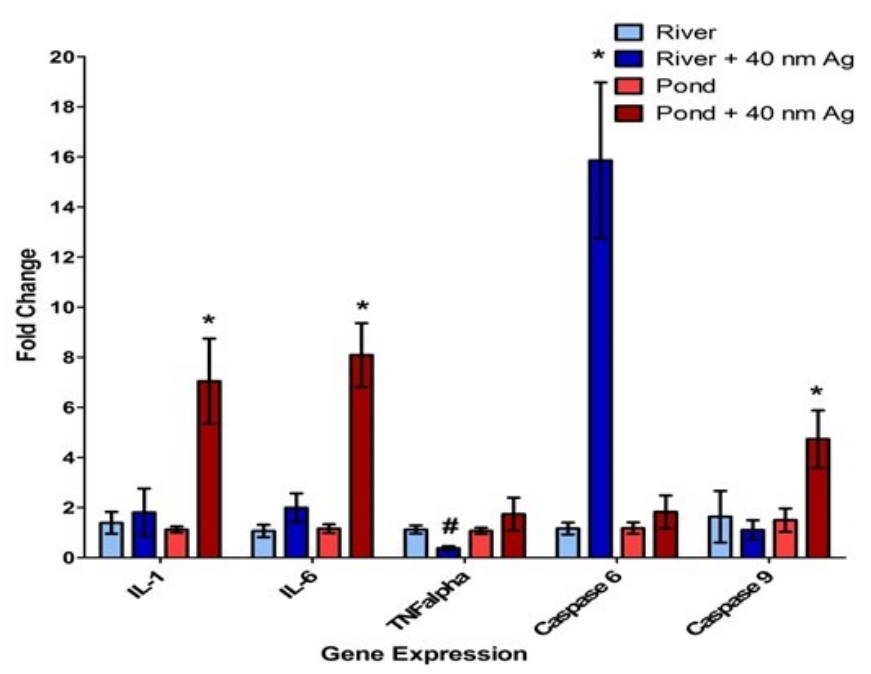

Figure 4. qPCR detection of gene expression in the kidneys of exposed and control fish. A comparison of inflammatory gene expression, and the expression of pro-apoptotic caspase genes were measured using reverse transcriptase qPCR to determine the impact of $40 \mathrm{~nm}$ AgNPs in the pond water (A) and river water (B). The control waters were normalized to 1 , and the fold change following AgNP exposure was determined using the delta-delta $\mathrm{Ct}$ method compared to similar control water with * indicating a significant upregulation and \# a significant downregulation of gene expression $(P<0.05)$ 
control (Figure 4). The caspase 9 gene was also activated in the AgNPexposed fish in the pond water, with a significant increase of 4.73-fold (+/- 1.04) in the AgNP fish over the pond water control (Figure 4). There were no significant changes in the expression of the TNFalpha or caspase 6 genes in the kidneys of the AgNP-treated pond water fish compared to the fish from the control pond water (Figure 4).

\section{Microbiota changes by AgNPs}

Following quality filtering and removal of chimeric and singleton sequences, there were 487,314 sequences remaining in the dataset that were assigned to 894 OTUs at $97 \%$ similarity. The OTUs represented 16 different bacterial phyla. The dominant phyla from pond samples were Proteobacteria (50\% of sequences), Firmicutes (22\%), and Actinobacteria (24\%), while the dominant phyla from river samples were Proteobacteria (58\%), Firmicutes (10\%), Bacteriodetes (9\%), and Actinobacteria (17\%) (Figure 5). After addition of AgNPs to zebrafish in pond water, the relative abundance of Actinobacteria decreased from $34 \%$ to $15 \%$, while Proteobacteria increased in relative abundance from

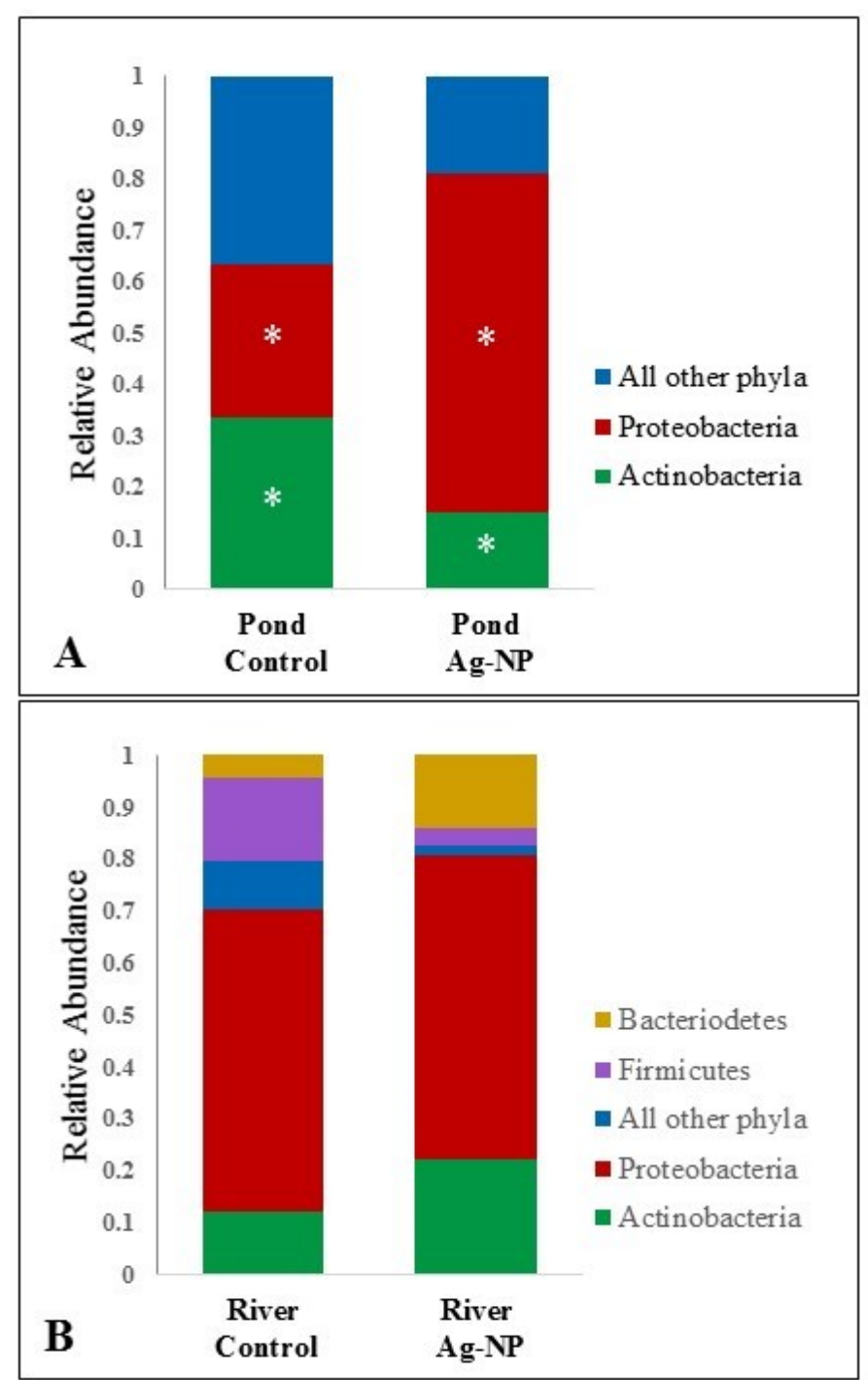

Figure 5. Microbial phyla from the skin and intestines of zebrafish. A comparison of bacterial phyla was performed using NGS to determine the impact of $40 \mathrm{~nm}$ AgNPs in the pond water (A) and river water (B). The AgNP exposed waters were compared only with their control with * indicating a significant change in microbial phyla $(P<0.05)$
$30 \%$ to $66 \%$ (nonparametric t-test, $P<0.05$ and $P<0.02$, respectively), (Figure 5A). Much of the increase in Proteobacteria following AgNP treatment was due to expansion of the genus Acidovorax from $10 \%$ to $34 \%$ of total sequence count (non-parametric t-test $P<0.03$ ). While exposure to AgNPs changed the community composition, it did not significantly alter the Shannon species diversity index or Chaol species richness measures. There were no significant changes in the bacterial flora between the control and AgNP groups from fish in the river water (Figure 5B).

\section{Discussion}

Although nanotechnology is an exciting field of research with many promising benefits, there has been some concern about environmental exposure following synthesis and use [16]. It is not well understood how stable the AgNPs are in the environment, and whether they remain complexed to soil particulates or accumulate in biological systems [16]. Due to salinity impacts it is estimated that freshwater fish are more susceptible to AgNP toxicology [17], but as demonstrated here there can be variation in the reactivity of AgNPs in freshwater. It has been previously demonstrated that dispersion media dramatically impacts AgNP stability [18]. Salts in the solution, $\mathrm{pH}$, and other impurities can impact the rate of agglomeration, thus impacting size, as well as the dissolution of nanoparticles into silver ions [18]. However, little has shown how these changes impact uptake into a host system and overall toxicity. Here we used two different environmental water sources and compared the impacts of the environmental water chemistry on AgNPs compared to nanoparticles dispersed in deionized $\mathrm{H}_{2} \mathrm{O}$. The differences in the water chemistry impacted the AgNPs, which contributed to a differential toxicity observed in zebrafish exposed to the two water sources.

Using a commercial water chemistry strip, it was determined that the water from a local river and a local pond had many differences, and these differences impacted the AgNP stability and toxicity. When $40 \mathrm{~nm}$ Biopure AgNP nanoparticles were added to the river water for 24 hours, the size increased to $188+/-9.4 \mathrm{~nm}$ and the $410 \mathrm{UV}$ Vis peak declined from 1.2 to 0.89 absorbance units (AU), suggesting an agglomeration and dissolution of the nanoparticle into silver ions. Following a 24-hour exposure of river water to AgNPs, 16 zebrafish were added to the water for 24 hours. After the 24-hours 4 of the zebrafish died, resulting in a $75 \%$ survival. The particles were more stable in the pond water, and no fish died. There is a discrepancy in studies regarding the lethality of AgNPs in aquatic organisms [16], and these discrepancies are likely largely due to water chemistry. ICPMS indicated that there was $250+/-3.47 \mathrm{ng}$ of silver in the kidneys of the fish, which was not characterized as ions or intact particles. Although the gills are often hypothesized as being the point of AgNP entry $[19,20]$, the kidneys were selected for toxicity measurements since they were demonstrated in a previous publication to have been a site for AgNP accumulation [6], which makes sense since they are involved in filtration and excretion. In response to the silver exposure, a $15.86+/-4.35$ upregulation of the caspase 6 gene was observed, and a $1.6+/-0.25$ fold decrease in TNF-alpha gene expression occurred. Upregulation of caspase 6 indicates an apoptotic response [21], but since caspase 9 was not upregulated it is not likely due to mitochondrial dysfunction [22]. Direct activation of caspase 6 is most often due to extrinsic factors such as DNA damage or death ligands binding to extracellular death domains [23]. The decline in TNF-alpha production suggests that the immune system is being modulated and could implicate poor macrophage function [23]. In addition to a cellular host impact, a change in the microbiota of the zebrafish was observed following 24-hour exposure to AgNP-treated river water. There were 
increases in the percentage of Actinobacteria and Bacteriodetes, and a decrease in Firmicutes and other non-dominant phyla, although none of these changes were statistically significant. Greater than $80 \%$ of vertebrate gastrointestinal (GI) microbiota is from species found within three phyla: Actinbacteria, Bacteroidetes, and Firmicutes [24]. Actinobacteria are one of the largest bacterial phyla found ubiquitously throughout the aquatic ecosystem [25]. They include both pathogens and GI commensals, such as the Bifidobacterium spp [25]. Although it is hard to discern any contributions due to the diversity of this phylum and the lack of significance, an increase in pathogenic bacteria or a disruption of GI microbiota can both lead to increased susceptibility to toxic compounds. Bacteroidetes and Firmicutes are frequently associated with being GI commensals, and their ratios can impact overall GI health [24]. A decreased Firmicutes:Bacteroidetes ratio (increased Bacteroidetes, increased Firmicutes) is indicative of weight loss [24], which too can cause an exacerbation of toxicology.

The pond water was obtained from a small, stand-alone pond with no stream or river influx. There were negligible nitrates and no detectable nitrites. The hardness was half that of the river (150 ppm) and the total alkalinity was nearly half that of the river $(180 \mathrm{ppm})$. These factors contributed to the lower $\mathrm{pH}$ of 7.4. The chlorine level was the same of $0.5 \mathrm{ppm}$. When $40 \mathrm{~nm}$ Biopure AgNPs were added to the pond water for 24-hours, it resulted in a slightly larger and more variable particle size than that of the river $(247.8+/-81.6 \mathrm{~nm})$. The $\mathrm{UV}-\mathrm{V}$ is $410 \mathrm{~nm}$ peak was also more affected in this water source as the AU dropped to 0.39 indicating even more dissolution into ions. After the 24-hour incubation, 16 zebrafish were added to the water samples, but this time an $100 \%$ survival rate was observed. Again, the kidneys were utilized for silver uptake and toxicology assessment. Only $75+/-0.52 \mathrm{ng}$ of silver was detected in the kidney of the pond fish, which is markedly lower than the fish that were in the AgNP-exposed river water. Inflammation was observed in the kidneys of the pond water AgNP fish with upregulation of both IL-1beta and IL-6 gene expression, suggesting that possibly an immune response is beneficial at preventing silver toxicity. An upregulation in caspase gene activity was also observed, but this time in caspase 9 , which suggests an intrinsic activation of apoptosis through mitochondrial dysfunction [22]. For the fish in the pond water samples, a significant difference in microbial flora of the fish was observed between the silver and non-silver treated pond water samples (Figure 5A). There was a significant increase in the bacterial phylum Proteobacteria, and a significant decrease in Actinobacteria (Figure 5A). Proteobacteria are comprised largely of pathogenic bacteria, which could perhaps be activating the immune response for clearance of both the bacteria and the nanoparticles [25]. However, it is far more likely that the silver nanoparticles are killing off some of the fish's normal microbiota, leaving it vulnerable to inhabitation by normal Proteobacteria members of the pond water and sediment [25]. The genera that demonstrated significant increases in the AgNP-treated pond water were Stenotrophomonas, Pseudomonas, Citrobacter, Variovorax, and Sphingomonas, all of which are found in soils or associated with plants [26]. This sample was suspected to dissolve into more silver ions, and other environmental toxicity studies have demonstrated a shift to a predominant Gram negative bacterial population, such as the Proteobacteria, following exposure to ionic liquids [27].

Most initial studies on AgNP toxicity were performed in rodents, but more recently researchers have begun to examine the effects on aquatic models, such as the zebrafish [16]. Zebrafish studies conclude that the synthesis methods, water chemistry, and overall ionic strength are some of the most important factors influencing environmental toxicology [28]. Here we demonstrate the importance of water chemistry on the uptake, toxicity, and microbial impact of AgNPs, and conclude that more studies need to be addressing the overall environmental impacts of these particles since they are being utilized in more consumer products and will likely end up in environmental waters in higher concentrations.

\section{Funding Sources}

We would like to thank the College of Science and Technology of Tarleton State University for the funds to complete this project.

\section{References}

1. Cox A, Venkatachalam P, Sahi S, Sharma N (2016) Silver and titanium dioxide nanoparticle toxicity in plants: A review of current research. Plant Physiol and Biochem 110: 33-49.

2. Duran N, Duran M, de Jesus MB, Seabra AB, Favaro WJ, et al. (2016) Silver nanoparticles: A new view on mechanistic aspects on antimicrobial activity. Nanomed: Nanotechnol, Biol, and Med 12: 789-799.

3. Juganson K, Mortimer M, Ivask A, Pucciarelli S, Miceli C, et al. (2017) Mechanism of toxic action of silver nanoparticles in the protozoan Tetrahymena thermophila: From gene expression to phenotypic events. Environ Pollution 225: 481-489.

4. Maurer LL, Meyer JN (2016) A systematic review of evidence for silver nanoparticleinduced mitochondrial toxicity. Environ Sci Nano 3: 311-322.

5. Haynes VN, Ward JE, Russell BJ, Agrios AG (2017) Photocatalytic effects of titanium dioxide nanoparticles on aquatic organisms-Current knowledge and suggestions for future research. Aquatic Toxicol 185: 138-148.

6. Speshock JL, Elrod N, Sadoski DK, Maurer E, Braydich-Stolle LK, et al. (2016) Differential organ toxicity in the adult zebra fish following exposure to acute sub-lethal doses of $10 \mathrm{~nm}$ silver nanoparticles. Front Nanosci Nanotech 2: 114-120.

7. Livak KJ, Schmittgen TD (2001) Analysis of relative gene expression data using realtime quantitative PCR and the 2(-Delta Delta C(T)) Method. Methods 25: 402-408.

8. Brady JA, Faske JB, Castañeda-Gill JM, King JL, Mitchell FL (2011) High-throughpu DNA isolation method for detection of Xylella fastidiosa in plant and insect samples. $J$ Microbiol Meth 86: 310-302.

9. Herlemann DPR, Labrenz M, Jurgens K, Bertilsson S, Waniek JJ, et al. (2011) Transitions in bacterial communities along the $2000 \mathrm{~km}$ salinity gradient of the baltic sea. ISME J 5:1571-1579

10. https://support.illumina.com/content/dam/illuminasupport/documents/documentation/ chemistry_documentation/16s/16s-metagenomic-library-prep-guide-15044223-b.pdf

11. Caporaso JG, Kuczynski J, Stombaugh J, Bittinger K, Bushman FD, et al. (2010) Qiime allows analysis of high-throughput community sequencing data. Nat Methods 7: 335-336.

12. Edgar RC (2010) Search and clustering orders of magnitude faster than blast. Bioinformatics 26: 2460-2461

13. DeSantis TZ, Hugenholtz P, Larsen N, Rojas M, Brodie EL, et al. (2006) Greengenes, a chimera-checked 16s rrna gene database and workbench compatible with arb. Appl Environ Microbiol 72: 5069-5072.

14. Cole JR, Wang Q, Cardenas E, Fish J, Chai B, et al. (2009) The ribosomal database project: Improved alignments and new tools for rrna analysis. Nucleic Acids Res 37: 141-145.

15. Lozupone C, Knight R (2005) Unifrac: A new phylogenetic method for comparing microbial communities. Appl Environ Microbiol 71: 8228-8235.

16. Speshock J (2018) Review of the toxicological effects of silver nanomaterials on the model aquatic organism Danio rerio. Front Nanosci Nanotech 4: 1-4.

17. van Aerle R (2013) Molecular mechanisms of toxicity of silver nanoparticles in zebrafish embryos. Environ Sci Technol 47: 8005-8014.

18. Gebauer JS, Treuel (2011) Influence of individual ionic components on the agglomeration kinetics of silver nanoparticles. J Coll and Interf Sci 354: 546-554.

19. Bilberg K, Hovgaard MB, Besenbacher F, Baatrup E (2012) In vivo toxicity of silver nanoparticles and silver ions in zebrafish (Danio rerio). J Toxicol 2012: 293784.

20. Osborne OJ, Lin S, Gao J, Bonzongo JC, Barber DS (2008) Effects of particle composition and species on toxicity of metallic nanomaterials in aquatic organisms. Environ Toxicol and Chem 27: 1972-1978. 
Speshock J (2020) Environmental water chemistry impacts silver nanoparticle characteristics, interactions with microbiota, and mechanisms of toxicity

21. Slee EA, Adrain C, Martin SJ (2001) Executioner caspase-3, -6, and -7 perform distinct, non-redundant roles during the demolition phase of apoptosis. $J$ Biol Chem 276: $7320-7326$

22. Haken R (1998) Differential requirement for caspase 9 in apoptotic pathways in vivo. Cell 94: 339-352.

23. Parameswaran N (2010) Tumor necrosis factor- $\alpha$ signaling in macrophages. Crit Rev Euk Gene Express 20: 87-103.

24. Mariat D (2009) The Firmicutes/Bacteroidetes ratio of the human microbiota changes with age. BMC Microbiol 9: 123-128.
25. Barka EA (2015) Taxonomy, physiology, and natural products of the Actinobacteria. Microbiol Mol Biol Rev 80: 1-43.

26. Llado S, Lopez-Mondejar R, Baldrian P (2017) Forest soil bacteria: Diversity, involvement in ecosystem processes, and response to global change. Microbiol and Mol Biol Rev 81: e00063-16.

27. Sydow M (2018) Biodiversity of soil bateria exposed to sub-lethal concentrations of phosphonium-based ionic liquids: Effects of toxicity and biodegradation. Ecotoxicol Environ Saf 147: 157-164.

28. Kim KT, Truong L, Wehmas L, Tanguay RL (2013) Sivler nanoparticle toxicity in the embryonic zebrafish is governed by particle dispersion and ionic environment. Nanotech 24: 115101.

Copyright: (C2020 Speshock J. This is an open-access article distributed under the terms of the Creative Commons Attribution License, which permits unrestricted use, distribution, and reproduction in any medium, provided the original author and source are credited. 